\title{
Estimation of relative water content in a sub-polar glacier using surface-penetration radar
}

\author{
Svein-Erik Hamran, "' Eldar Aarholt \\ Environmental Surveillance Technology Programme, PFM, P.O. Box 89, 2001 Lillestrom, Norway \\ Jon Ove Hagen \\ Department of Physical Geography, University of Oslo, P.O. Box 1042 Blindern, 0316 Oslo, Norway \\ PER Mo \\ Norwegian Institute of Technology, 7034 Trondheim, Norway
}

\begin{abstract}
Radar-sounding data taken at Uvérsbreen glacier, Svalbard, are used to estimate the relative water content of the temperate part of the glacier. The relative change in water content is estimated from the strength of the backscattered radar energy without knowing the size of the scatterers. The main findings are that the water content is increasing gradually with depth below the cold/temperate interface. The water content is on average changing by a factor of five in the ice, with the exception of some bright spots believed to stem from larger water moulins. The general trend is also that the water content is increasing with lower altitude. The applied technique may be a useful method for large-scale mapping of the relative watercontent variation in temperate and polythermal glaciers.
\end{abstract}

\section{INTRODUCTION}

Mapping the three-dimensional distribution of water content in a temperate glacier is a difficult task. In situ measurements made using instruments lowered in boreholes are time-consuming and thus limit the amount of data that can be collected. One alternative is to measure the radar-wave velocity between two boreholes or from a borehole to the surface (Kotlyakov and Macheret, 1987; Macheret and others, 1993). This yields an integrated value of the dielectric constant which can be used to estimate the average water content along the radar wavepath. The disadvantage, again, is the need to use boreholes; making a map over an entire glacier is a timeconsuming task. The only reasonably rapid alternative is to use surface-based radar measurements; backscattered energy from water inclusions is used to map the water content (Bamber, 1988, 1989). The problem, however, is that the size of the inhomogeneities is unknown, making proper modeling difficult. If the scattering inhomogeneities were much smaller than the radar wavelength, Rayleigh scattering theory could be used. But this is not the case: the size of the scatterer is of the same order as the radar wavelength. If the water inclusions were assumed

\footnotetext{
* Present Address: Norwegian Defence Research Establishment (FFI), Division for Electronics, PO Box 25, N-2007 Kjeller, Norway
}

spherical, Mie scattering theory could be used (Bamber, 1987); the problem remains, however, that one has to know the size of the water inclusions.

Another major obstacle in interpreting and inverting ground-penetrating radar data is lack of knowledge of the antenna-to-medium coupling. When the radar is operated from a ground-based platform, antennas are positioned close to the ground and the radiation pattern is strongly perturbed by the air/medium interface. The antenna-toground coupling may also change as the radar is moved.

In short, it is difficult to model absolute scattering from free water in a temperate glacier. In this paper, our approach is to look only for relative changes in water content and not for the absolute water content. We show that the change in water content can be estimated from the backscattered radar energy without knowing the size of the scatterers. This technique can then be combined with one of the above-mentioned absolute calibrated techniques to get the true change in water content.

Radar measurements have been extensively used to map the temperate layer at glaciers at Svalbard Odegård and others, 1992; Hamran and Aarholt, 1993; Bjornsson and others, 1996). The radar data presented here were taken on Uvérsbreen, Svalbard, in May 1991 (Fig. 1).

\section{RADAR SYSTEM}

The radar system used to collect the data is a range-gated synthetic pulse system (Hamran and Aarholt, 1993; 


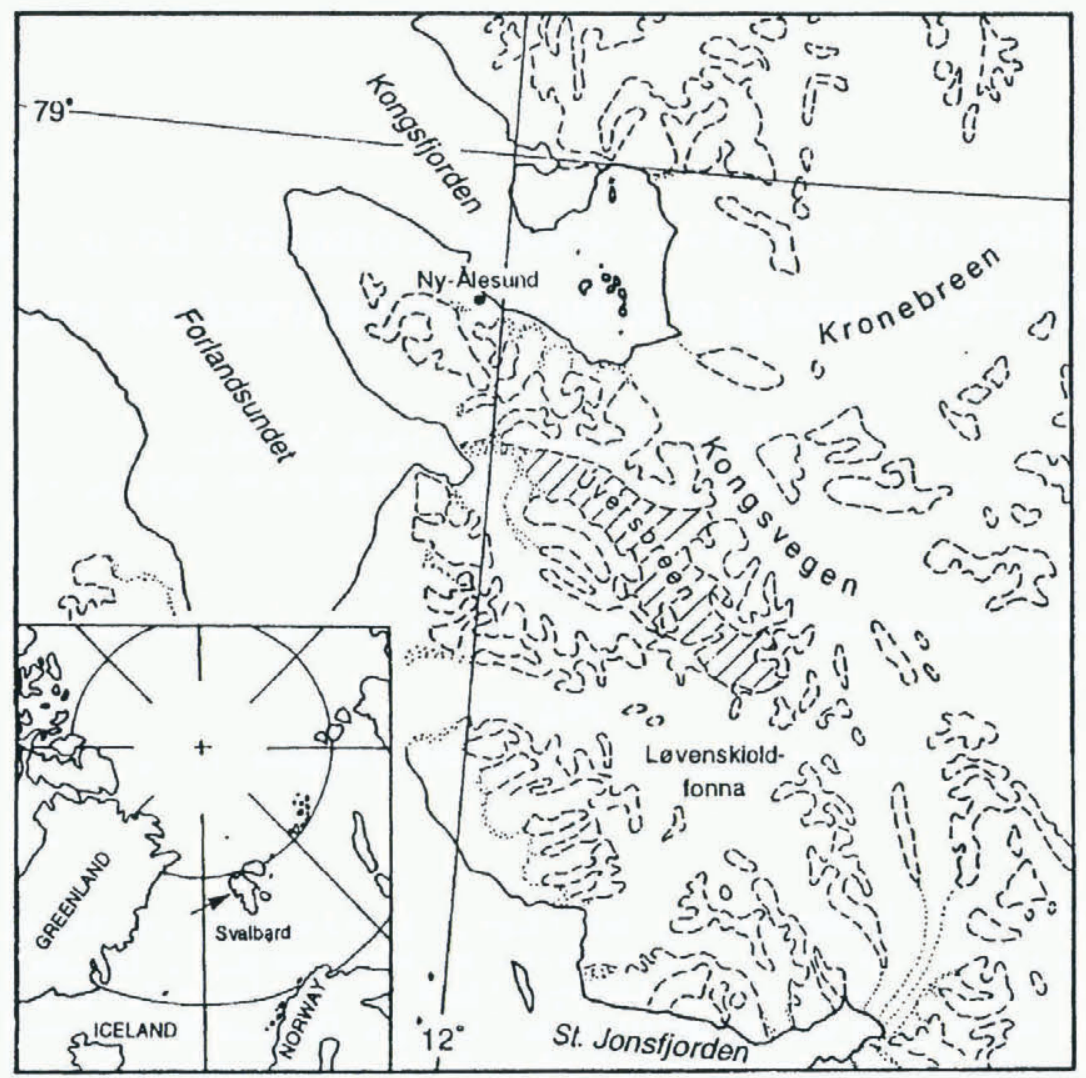

Fig. 1. Geographical position of Uvérsbreen.

Hamran and others, 1994). The operational-frequency bandwidth is large, $0.1 \mathrm{MHz}-3 \mathrm{GHz}$, covering the most interesting wavelengths used in glacier studies and subsurface applications. Depending on the antennas used, the glacier can be illuminated using a large spectrum of wavelengths. In this experiment, only one set of antennas was used and the spectrum was chosen to give optimal scattering from free water in the glacier at a sufficient depth resolution. Returns from 201 frequencies in the range $320-370 \mathrm{MHz}$ were collected every $8.4 \mathrm{~m}$ along the glacier. These were inverted to the time domain using an inverse Fourier transform with no further processing of the data.

\section{RADAR EQUATION}

The radar equation (Ulaby and others, 1981) gives the received power, $P_{\mathrm{r}}$ as:

$$
P_{\mathrm{r}}=\frac{P_{\mathrm{t}} G^{2} \lambda^{2} \sigma}{(4 \pi)^{3} R^{4}} \mathrm{e}^{-4 \alpha R}
$$

where $P_{\mathrm{t}}$ is the transmitting power, $G$ is the antenna gain, $\lambda$ is the radar wavelength in the ice, $\sigma$ is the scattering cross-section, $R$ is the depth and $\alpha$ is the attenuation coefficient due to absorption and scattering in the ice.

As mentioned, it is difficult to model the scattering cross-section due to lack of knowledge regarding the size and shape of the scatterers. We therefore make some simplifying assumptions to make it easier to invert the data. The first step is to assume single scattering, that is, we neglect interference between waves scattered by different water particles, and merely add the power scattered from the different particles (Ishimaru, 1978). The scattering cross-section in Equation (1) can be written as

$$
\sigma=\rho \sigma_{1} V
$$

where $\sigma_{1}$ is the scattering cross-section from one water particle and $\rho$ is the number of particles in the volume $V$. We need to take into account the fact that water inclusions do not all have the same size but have a size distribution (Ishimaru, 1978). Let $n(D, R) \mathrm{d} D$ be the number of particles per unit volume at position $R$ having a range of sizes between $D$ and $(D+\mathrm{d} D)$, and $\langle\sigma\rangle$ be the average scattering cross-section. Then the scattering cross-section should be replaced by

$$
\sigma=\int_{0}^{\infty} n(D, R) \sigma_{1}(D) V \mathrm{~d} D=\rho\langle\sigma\rangle V
$$

(Ishimaru, 1978). We now compare the received scattered power from two different parts of the glacier. The volume is limited by the antenna opening angle and the radar pulse length in the ice. Combining Equations (1) and (3) yields

$$
\frac{P_{\mathrm{r}}^{\prime}}{P_{\mathrm{r}}^{\prime \prime}}=\frac{R^{\prime \prime 4} \rho^{\prime}\left\langle\sigma^{\prime}\right\rangle V^{\prime}}{R^{\prime 4} \rho^{\prime \prime}\left\langle\sigma^{\prime \prime}\right\rangle V^{\prime \prime}} \mathrm{e}^{-4 \alpha\left(R^{\prime}-R^{\prime \prime}\right)} .
$$

The volume $V$ can be written in terms of the antenna gain, $G$, as (Bamber, 1988) 


$$
V=\frac{2 \pi R^{2} L}{G}
$$

where $L$ is the pulse length in the ice. Substituting Equation (5) into Equation (4) gives

$$
\rho^{\prime}=\rho^{\prime \prime} \frac{P_{\mathrm{r}}^{\prime} R^{\prime 2}\left\langle\sigma^{\prime \prime}\right\rangle}{P_{\mathrm{r}}^{\prime \prime} R^{\prime \prime 2}\left\langle\sigma^{\prime}\right\rangle} \mathrm{e}^{4 \alpha\left(R^{\prime}-R^{\prime \prime}\right)} .
$$

Assuming that a change in the water content in the glacier is the result of a change in the number of water particles and not of a change in particle size, then $\left\langle\sigma^{\prime}\right\rangle=\left\langle\sigma^{\prime \prime}\right\rangle$, and Equation (6) can be written as

$$
\rho^{\prime}=\rho^{\prime \prime} \frac{P_{\mathrm{r}}^{\prime} R^{\prime 2}}{P_{\mathrm{r}}^{\prime \prime} R^{\prime \prime 2}} \mathrm{e}^{4 \alpha\left(R^{\prime}-R^{\prime \prime}\right)} .
$$

Thus if $\rho^{\prime \prime}$ is known or is set equal to $100 \%$, and a loss value for $\alpha$ is assumed, then the relative water content in the glacier can be estimated using the received scattered power from different parts of the glacier.

If an in situ measurement or a radio-wave velocity measurement is done, and the absolute water content is measured at $\rho^{\prime \prime}$, then using Equation (7) a subsurface map of the water content in the glacier can be made. Equation (7) was used to make a subsurface map of the relative water content in Uvérsbreen.

\section{UVÉRSBREEN}

Uvérsbreen is on the northwest coast of Spitsbergen at $78.5^{\circ} \mathrm{N}, 12.3^{\circ} \mathrm{E}$ (Fig. 1). It is a typical valley glacier, with a well-defined catchment area. The length of the glacier is $20.5 \mathrm{~km}$ and its area is $63.5 \mathrm{~km}^{2}$. The glacier front ends on land, behind a large field of ice-cored end moraines. The glacier mass balance is not measured, but, based on measurements made on other glaciers in this part of Spitsbergen, the mass balance is probably negative and has been since about 1920. However, the glacier front has not retreated as much as other glacier fronts in this region.

Our radar sounding along the main axis of the glacier shows a maximum thickness of about $250 \mathrm{~m}$. In Figure 2 a longitudinal cross-profile of the glacier is shown including an interpretation of radar measurements giving the thickness and extension of the cold layer.

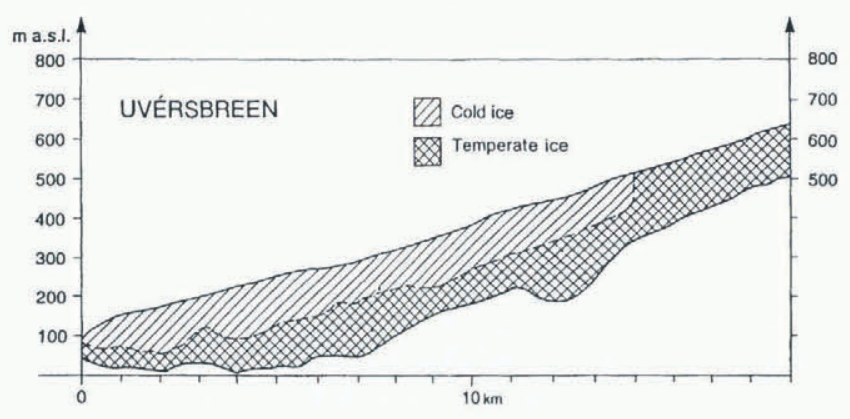

Fig. 2. Schematic diagram of Uversbreen showing the extent of the cold surface layer overlaying the temperate basal layer, derived from radar measurements.

\section{RESULTS AND DISCUSSION}

Figure 3 shows the radar data from a profile running along the center line of the glacier, with power displayed as a function of depth. It is clearly not possible to use a plane reflection model at the cold/warm ice interface; the scattering is incoherent and several point scatterers contribute to the signal strength. In the first $6 \mathrm{~km}$ of the profile, scattering from the bottom of the glacier can be seen. The signal strength of the scattering varies more than $35 \mathrm{~dB}$ in the radar image. The received power is strong close to the cold/temperate ice interface and decreases with depth.

Based on these data and Equation (7), the relative water content is calculated and shown in Figure 4. The image is pixel-averaged by summing over an area of 3 pixels by 3 pixels. The reference volume chosen is at $2.3 \mathrm{~km}$ distance and $110 \mathrm{~m}$ depth where strong scattering is seen. This volume shows a high signal strength and thus is expected to contain a high percentage of water. The attenuation coefficient given by Bamber (1987) is typically $4.0-4.5 \mathrm{~dB}$ per $100 \mathrm{~m}$ on Svalbard glaciers. Not clearly stated, this is interpreted as the average value for both cold and temperate ice. We have chosen the value $4.5 \mathrm{~dB}$ per $100 \mathrm{~m}$ as the attenuation in the temperate layer. The results clearly depend on the chosen value for the attenuation coefficient. The maximum thickness of temperate ice is in our case $100 \mathrm{~m}$, so if the chosen attenuation value is $0.5 \mathrm{~dB}$ wrong the estimated received power would be a maximum of $1 \mathrm{~dB}$ wrong. This gives a maximum error in the relative water content of about $25 \%$, less at shallower depths. The total absorption in the temperate layer is probably higher than the one estimated, due to scattering loss. If in situ measurements of the water content were performed at one or more locations, a better calibration of the absorption could be made.

The estimated water content is color-coded according to percentage, and the scaling is shown to the right of the image. On average, the water content is $10-50 \%$ of the reference value. The water content increases gradually from the cold/warm interface down to $10-20 \mathrm{~m}$ below the interface, and is almost constant from there to the bottom of the glacier. The bright red spots may be larger moulins or other parts of the englacial drainage system. The water content also increases with lower elevation. The exception is a bright spot at $10.5 \mathrm{~km}$. Point scatterers have a strong frequency dependence which could be used to study the size distribution of the water particles. This would improve the scattering model.

Radar soundings on other glaciers have shown that the temperature regime can be mapped since the interface between cold and temperate ice is readily detected (Odegaard and others, 1992; Hamran and Aarholt, 1993; Bjornsson and others, 1996). Our measurements show that Uvérsbreen is a typical sub-polar or polythermal glacier, where the accumulation area is temperate throughout and the ablation area is cold down to about $100 \mathrm{~m}$ depth. The glacier is temperate below this depth and thus contains free water. Meltwater from the surface of the accumulation area can penetrate down through the glacier in the accumulation area. In the ablation area, meltwater runs off at the ice surface. The 


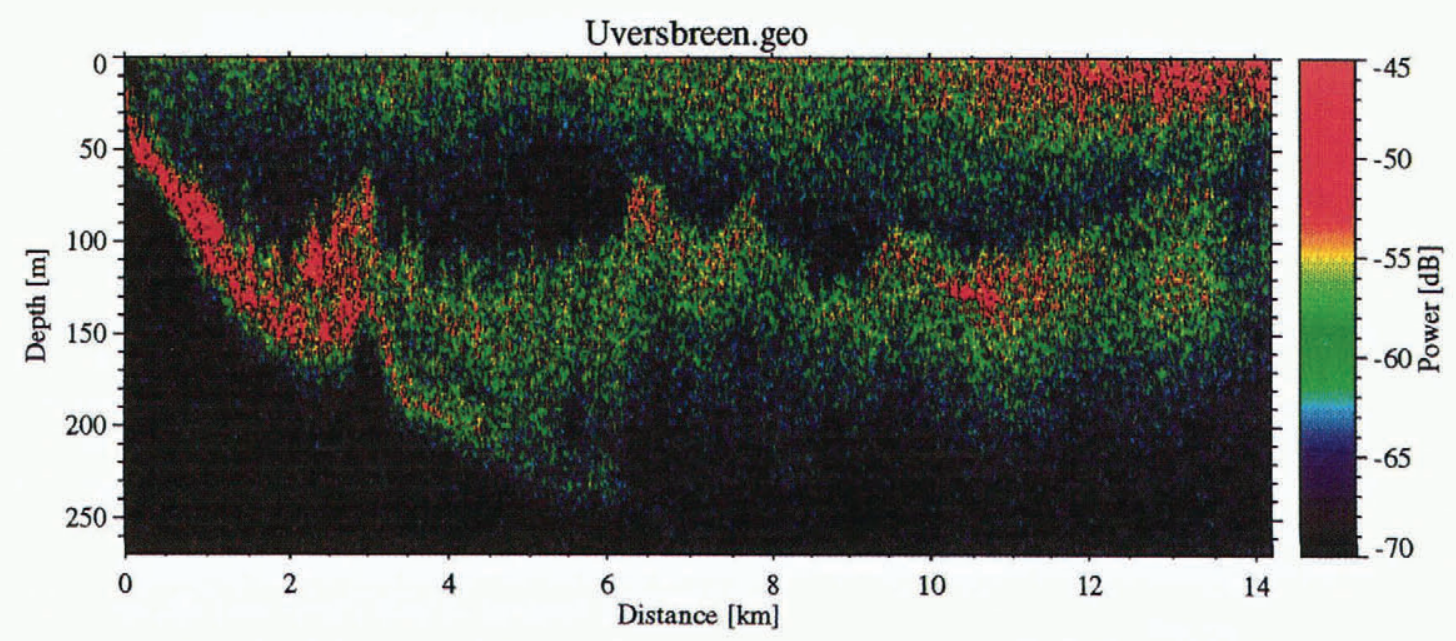

Fig. 3. Radar data from a profile running along the center line of the glacier, with power displayed as a function of depth.

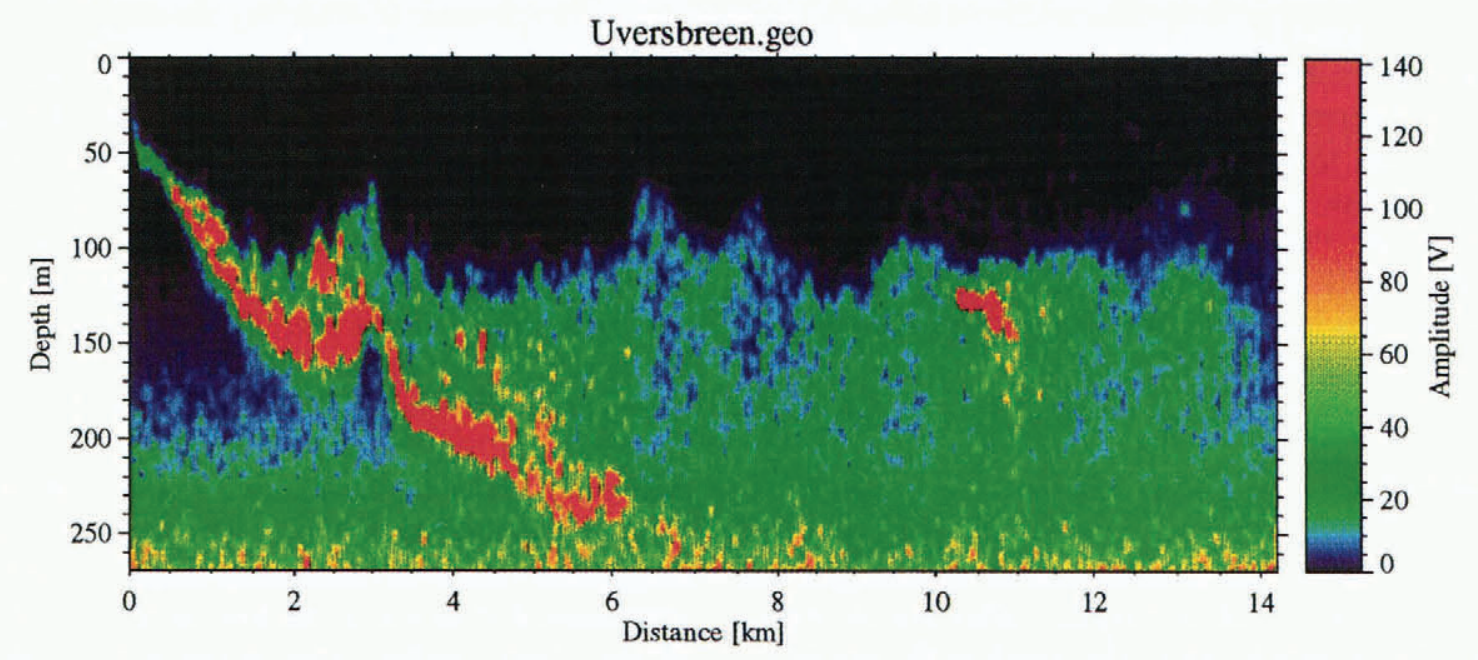

Fig. 4. The radar data in Figure 3 transformed using Equation (7) to give the relative water content at different positions on the glacier.

water drains into channels cut into the ice by the meltwater streams or into moulins or crevasses that lead the water into englacial channels. Through the cold part the water is concentrated in channels, and no free water exists outside these channels.

The radar experiments should be continued by a test throughout the year to see if it is possible to see any seasonal changes in the maximum water content. Observations in the partly water-filled moulins in some larger tidewater glaciers indicate that the water level is at its lowest in early autumn and at its highest in early spring. A water-level increase of almost $100 \mathrm{~m}$ during the winter has been observed in a moulin on Hansbreen, southern Spitsbergen (personal communication from J. Schroeder, 1992).

\section{CONGLUSIONS}

The technique under study shows a high potential for large-scale mapping of the water content in temperate and sub-polar glaciers. Combining the technique used here with in situ measurements of the water content, at different positions on the glacier, is a rapid and simple way of mapping the water content. Successive measurements over the year mapping the water content would give the seasonal variations. This could give important information for understanding both glacier hydrology and the surge process.

\section{ACKNOWLEDGEMENTS}

The authors are grateful for all the help given by $\mathrm{R}$. Hansen, J. Kohler and R. Engseth during the preparation of this paper.

\section{REFERENCES}

Bamber, J. L. 1987. Internal reflecting horizons in Spitsbergen glaciers. Ann. Glaciol., 9, 5-10.

Bamber, J. L. 1988. Enhanced radar scattering from water inclusions in ice. f. Glaciol., 34 (118), 293-296.

Bamber, J. L. 1989. Ice/bed interface and englacial properties of Svalbard ice masses deduced from airborne radio echo-sounding data. J. Glaciol., 35 (119), 30-37.

Björnsson, H. and 6 others. 1996. The thermal regime of sub-polar glaciers mapped by multi-frequency radio-echo sounding. \%. Glaciol., $42(140), 23-32$. 
Hamran and others: Relative water content in a sub-polar glacier

Hamran, S. -E. and E. Aarholt. 1993. Glacier study using wavenumber domain synthetic aperture radar. Radio Sci., 28 (4), 559-570.

Hamran, S. -E., D. T. Gjessing, J. Hjelmstad and E. Aarholt. 1994. Ground penetration synthetic pulse radar: dynamic range and modes of operation. 7. Appl. Phys., 33 (1985), 7-14.

Ishimaru, A. 1978. Wave propagation and scattering in random media. New York, Academic Press.

Kotlyakov, V. M. and Yu. Ya. Macheret. 1987. Radio echo-sounding of sub-polar glaciers in Svalbard: some problems and results of Soviet studies. Ann. Glaciol., 9, 151-159.
Macheret, Yu. Ya., M. Yu. Moskalevsky and E. V. Vasilenko. 1993. Velocity of radio waves in glaciers as an indicator of their hydrothermal state, structure and regime. f. Glaciol., 39(132), 373-384.

Odegård, R. S., S. -E. Hamran, P. H. Bø, B. Etzelmüller, G. Vatne and J.L. Sollid. 1992. Thermal regime of a valley glacier, Erikbreen, northern Spitsbergen. Polar Res., $11(2), 69-79$.

Ulaby, F. T., R. K. Moore and A. K. Fung. 1981. Microwave remote sensing: active and passive. Vol. 1. Microwave remote sensing fundamentals and radiometry. Reading, MA, Addison-Wesley Publishing Co.

MS received 8 . November 1995 and accepted in revised form 15 May 1996 